\title{
Electronic media use and sleep in children and adolescents in western countries: a systematic review
}

\author{
Lisbeth Lund, Ida Nielsen Sølvhøj, Dina Danielsen and Susan Andersen* (1)
}

\begin{abstract}
Background: Sleep is essential for child and adolescent health and well-being. There is an increasing interest in whether electronic media use affects children and young adolescents' sleep. Prior reviews have focused on a school-aged population. Moreover, it is crucial that research continuously addresses the processes of technology and media use and the implication on sleep. This systematic review examines the evidence of electronic media use related to sleep among $0-15$-year-olds.

Methods: Searches were carried out in four databases (CINAHL, Web of Science, EMBASE, and Medline). Inclusion criteria included age $\leq 15$ years, and intervention, cohort, or cross-sectional studies from western countries. Methodological quality was rated using the Quality Assessment Tool for Quantitative Studies by two independent reviewers. Data was extracted using a standardized data extraction form. Synthesis was done by summarizing results across studies by age groups of $0-5,6-12$, and $13-15$ years within four sleep domains: Bedtime and sleep onset; Sleep quality; Sleep duration; Daytime tiredness.
\end{abstract}

Results: The search identified 10,719 unique studies, of which 109 fulfilled inclusion and exclusion criteria and were assessed for methodological quality. In total, 49 studies were included in the review. The study designs were randomized controlled trials $(n=3)$, quasi-experimental studies $(n=2)$, prospective cohort studies $(n=15)$, and cross-sectional studies $(n=29)$. Evidence for an association between electronic media use and sleep duration was identified, with stronger evidence for 6-15-years-olds than 0-5-year-olds. The evidence for a relationship between electronic media use and other sleep outcomes was more inconclusive. However, for 6-12-year-old children, there was evidence for associations of electronic media use with delayed bedtime and poor sleep quality. For 13-15-yearolds, there was evidence for associations between screen time and problems falling asleep, and between social media use and poor sleep quality.

Conclusions: Overall, electronic media use was generally associated with shorter sleep duration in children and adolescents. Studies with stronger research design and of higher quality are needed to draw solid conclusions about electronic media's impact on other sleep outcomes. Public awareness and interventions could be promoted about the potential negative impact on children's sleep of electronic media devices that are used excessively and close to bedtime.

Keywords: Systematic review, Child, Adolescent, Telecommunications, Sleep

\footnotetext{
* Correspondence: suan@sdu.dk

National Institute of Public Health, University of Southern Denmark,

Studiestræde 6, DK-1455 Copenhagen, Denmark
}

(C) The Author(s). 2021 Open Access This article is licensed under a Creative Commons Attribution 4.0 International License, which permits use, sharing, adaptation, distribution and reproduction in any medium or format, as long as you give appropriate credit to the original author(s) and the source, provide a link to the Creative Commons licence, and indicate if changes were made. The images or other third party material in this article are included in the article's Creative Commons licence, unless indicated otherwise in a credit line to the material. If material is not included in the article's Creative Commons licence and your intended use is not permitted by statutory regulation or exceeds the permitted use, you will need to obtain permission directly from the copyright holder. To view a copy of this licence, visit http://creativecommons.org/licenses/by/4.0/ The Creative Commons Public Domain Dedication waiver (http://creativecommons.org/publicdomain/zero/1.0/) applies to the data made available in this article, unless otherwise stated in a credit line to the data. 


\section{Background}

Sleep has a major impact on the health and well-being of children and adolescents. Sleep is vital for development and learning ability, and insufficient sleep over an extended period can have long-term physical and psychological health implications [1]. Physiological and psychological changes that emerge in childhood and youth may impact negatively on sleep, but poor sleep is arguably also related to, or compounded by, external factors such as early school start times, environmental conditions in the bedroom (e.g. noise, high temperature or too much light) and the availability of electronic media [1-3]. Over the last few decades, a major lifestyle change has happened due to the incorporation of electronic media device use into people's daily life. Electronic media has become a core part of young people's lives and children today are growing up surrounded by electronic media devices. Studies demonstrate that most children, even as young as four months of age, have experience with using electronic media devices, although electronic media consumption is largest among older teenagers [4]. Media devices have become an integral part of children's development environment and a prevalent mode of communication among adolescents [5]. Although electronic media is widely accepted and accessible in the home environment, there is yet only a limited understanding of how access to and use of electronic media may impact the sleep patterns of children and adolescents.

Technology is continuously evolving, and the way electronic media devices are used in everyday life may change over time. Although previous systematic reviews and meta-analyses have established a correlation between media use and sleep [6-8], it is crucial that research continuously addresses the processes of technology and media use and its implication on children and adolescents' sleep patterns. Therefore, reviews that include the newest types of electronic media devices and technological trends are needed. Moreover, previous reviews on this subject focus mainly on an older paediatric target group, and there is limited knowledge about the evidence of electronic media devices and the impact on sleep among pre-schoolers [9].

The aim of this study was to systematically review the literature on the impact of using electronic media on sleep in children and adolescents. The population was preschool children, school-age children up to 12-years-old and young adolescents up to 15 years old. The exposure was access to and use of electronic media devices, and the outcomes were bedtime and sleep onset, sleep quality during nighttime, sleep duration and daytime tiredness. The intention was to inform policy and practice and to highlight what further research is needed on this topic.

\section{Methods}

A narrative method was applied to synthesis the data on the association between electronic media use and sleep among children and adolescents.

\section{Eligibility criteria}

We included studies which fulfilled the following eligibility criteria: (1) Assessed the associations between the use of or access to electronic media devices and sleep, i.e. delayed bedtime, sleep onset latency, sleep quality during night-time, sleep duration and daytime tiredness; (2) Published in English between January 1, 2009 and August 31, 2019; (3) From western countries; (4) Examined children and adolescents between 0 and 15.9 years of age without any diagnoses/diseases. Electronic media devices were defined as mobile phones, televisions, touchscreens/tablets, computers, or video game consoles. The exclusion criteria were apps intended to treat sleep disorders, or problems (e.g., sleep apnoea), and studies examining electromagnetic radiation.

\section{Data sources and search strategy}

The PICo model was used to generate the search strategy, and the search strategy was divided into three search blocks: P (Patient / Problem / Population), I (Phenomenon of interest), and Co (Context). The population was children and adolescents aged 0-15; the field of interest was electronic media devices, and the context was before sleep (bedtime and sleep onset), during sleep (sleep quality during nighttime), and after sleep (sleep duration and daytime tiredness). We performed a systematic search in four databases: CINAHL, EMBASE, Web of Science, and Medline based on keywords (subject headings/MeSH terms) and free text searches (title, keywords, text). The search terms and syntax included relevant synonyms for the search terms adolescents/children (e.g. minor, teenager), electronic media devices (e.g. cell phone, screen), and sleep (e.g. sleep latency, bedtime routine) (see Supplementary eTable 1 for the full search in each database). In addition, we included previous reviews to identify relevant studies.

\section{Study selection; screening, quality assessment, and data extraction}

Title and abstracts identified were screened for eligibility, and full texts of potentially eligible articles were read and assessed by two reviewers (LL and INS) independently. Discordance regarding inclusion was resolved through discussion. Two reviewers (LL and SA) independently assessed the methodological quality of the included quantitative studies. To ensure consistency in the quality assessments, meetings were held on an ongoing basis, focusing on inter-rater reliability. The methodological quality of the quantitative articles was assessed using the Effective Public Health Practice Project (EPHP P) assessment tool [10], based on the following five components: selection bias, study design, confounders, data collection methods, and withdrawal/dropouts. The EPHPP covers any quantitative study design, it is 
developed for use within public health and has been found to have psychometric properties as good as the Cochrane risk-of-bias tool. Each study was rated as high, moderate, or low quality. Low-quality studies were excluded to ensure moderate evidence. We extracted the data using a standardized data extraction form. It included country of study, age, sex, study design, sleep outcomes, exposure (electronic media device measures) and reported associations. Synthesis was done by summarizing results and conclusions across studies grouped by age groups of $0-5.9$ years, $6-12.9$ years, and $13-15.9$ years within four sleep domains: Bedtime and sleep onset; Sleep quality; Sleep duration; Daytime tiredness. If a study included data on ages overlapping the defined age categories, we used the mean age to allocate the study to an age category. A few studies $(n=2)$ included a large age span. However, these studies had performed analyses by subgroups of age corresponding to our categories.

\section{Results}

A total of 446 full texts were reviewed, of which 338 were excluded (Fig. 1). We identified 7 qualitative studies of which 3 studies received high- or moderate-quality assessments. These studies focused on factors (e.g. the role of parents) that facilitate the accessibility and acceptability of electronic media use in relation to sleep, and not on how electronic media use might impact sleep in children and adolescents. The results from the qualitative studies will be reported in a separate paper given the focus on quantitative studies in this systematic review.. In total, 52 quantitative studies were not included because they received a low-quality assessment rating as a result of a range of methodological issues: weakness in study design, a small percentage of responses, not controlling for confounders, and not reporting validity and reliability of measures used. Of the 49 included quantitative studies, four studies received a high-quality assessment rating, and 45 studies received a moderate-quality assessment rating. Of the included studies, 18 were conducted in North America (USA and Canada) [11-28], 23 in Europe (England, Finland, Sweden, Holland, Switzerland, France, Italy, Spain, and Germany) [29-51], five in Australia and New Zealand [52-56], and three studies combined several western countries [57-59]. There were three randomized controlled trials (RCTs) $[27,28,50]$, two quasi-experimental studies $[26,51], 15$ prospective cohort studies [12, 16, 19, 24, 30, 32, 35, 37, $40,42,45,47,48,52,55]$, and 29 cross-sectional studies $[11,13-15,17,18,20-23,25,29,31,33,34,36,38,39$, $41,43,44,46,49,53,54,56-59$ ] (see Table 1 for chacteristics of the included studies). The majority of the included studies adjusted for all or most of the following confounders: ethnicity, age/grade, sex, and socioeconomic status (e.g., parental education and/or parental occupation). Some studies also included health factors such as BMI, psychological symptoms, and physical activities. Detailed descriptions of study design and results are available in Supplementary eTables 2-4, and references on excluded studies in Supplementary eTable 5.

\section{Electronic media use among children aged 0-5}

Table 2 summarizes the results of the 13 studies [11-15, 27, 29-32, 52, 56, 57] included for 0-5-year-olds.

\section{Bedtime and sleep onset}

Five studies analysed the association between electronic media use and late bedtime and/or sleep onset [11, 13 , 14, 29, 52]. Three studies examined tablet use and found an association of tablet use (both general and at bedtime) with delayed bedtime or sleep onset latency [11, $13,14]$. There were two studies on television viewing before bedtime and both found an association with delayed bedtime or sleep onset latency $[11,13]$. Two studies estimated the association between mobile phone use and delayed bedtime or sleep onset latency with one finding a positive association for the use of mobile phones at night-time [14] and one finding no association [11]. The association between gaming and delayed bedtime or sleep onset latency was examined in three studies [11, $13,14]$ with one study reporting an association [13]. One longitudinal study examined screen time in 2-yearolds and found a positive association with sleep onset latency in 5-year-olds [52]. No evidence was found for an association between computer/Internet use and delayed bedtime/sleep onset $[11,13,14]$. One study found an association between the presence of a television in the bedroom and delayed bedtimes on weekdays, but not on weekends [13].

\section{Sleep quality}

There was no evidence for television viewing or use of touchscreens [29], while there was inconsistent evidence for general screen time use $[15,52,56]$ on night awakenings or sleep disturbances. An intervention study showed that promotion of prosocial content on electronic media reduced sleep problems [27].

\section{Sleep duration}

Regarding sleep duration, three studies showed that overall screen time was associated with shorter sleep duration $[15,52,57]$, and six studies showed this concerning television viewing $[11-13,30-32]$ and three studies concerning use of touch screen or tablet [11, 14, 29]. Lack of association was found for the use of mobile phones $[11,14]$, video gaming $[11,13,14]$ and computers or Internet $[11,13,14]$. Inconsistent evidence was found for the presence of electronic media devices in the bedroom [12, 13]; for ethnic minority children, television 


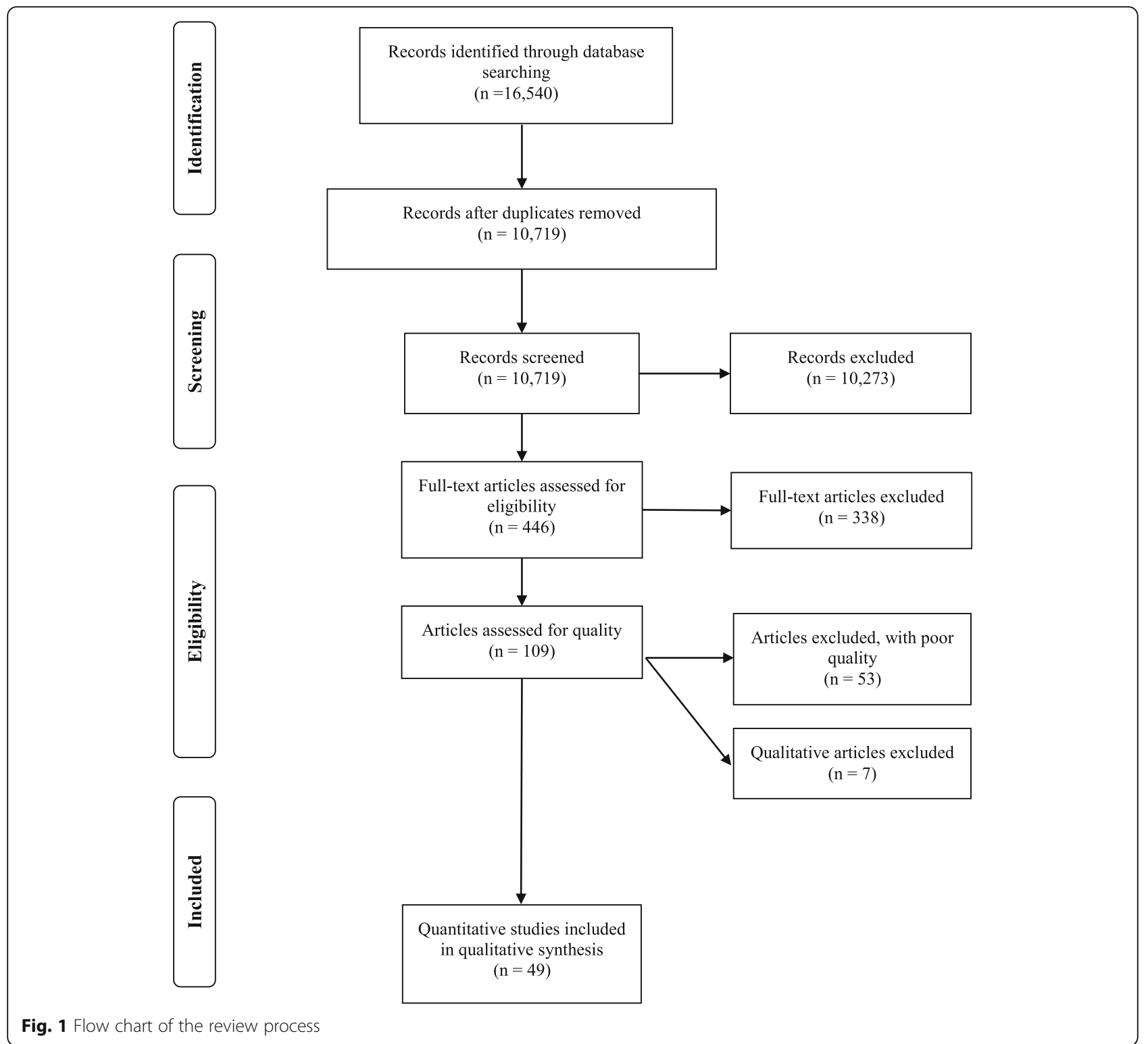

in the bedroom among 4-year-olds was associated with 32 fewer minutes of sleep per day at age 7 [12].

\section{Daytime sleep duration}

Three studies found associations between television viewing and longer naps [11, 13, 29], while inconsistent evidence was found for the use of mobile phones $[11,14]$ and touch screen or tablet $[11,14,29]$. No evidence was found for gaming, computer or internet use, and the presence of electronic media devices in the bedroom [11, 13, 14].

\section{Electronic media use among children aged 6-12}

Table 3 summarizes the results for the 15 studies [15$21,28,30,33-37,53]$ included for the age range $6-12$.

\section{Bedtime and sleep onset}

Six studies analysed the association between electronic media use and late bedtime and/or sleep onset $[18,33$, 35-37, 53]. Five of the studies found an association. Some studies only found an association when stratified by specific variables such as weekends/weekdays. For example, Mireku et al. (2019) showed that use of screenbased media device in the last hour before bedtime was associated with 1.44 times the odds of delayed sleep onset on weekends, but no association was found on weekdays [36]. Two studies assessed the association between video gaming and sleep onset $[18,33]$. Arora et al. (2014) showed that high frequency of gaming at bedtime was associated with a 6.2 min prolonged sleep onset on weekdays [33]. Falbe et al. (2015) reported that each hour per day of gaming was associated with a $9.8 \mathrm{~min}$ 
Table 1 Study characteristics of the 49 included studies in the systematic review

\begin{tabular}{|c|c|}
\hline & n (\%) \\
\hline \multicolumn{2}{|l|}{ Geographic location } \\
\hline North America & $18(37)$ \\
\hline Europe & $23(47)$ \\
\hline Australia and New Zealand & $5(10)$ \\
\hline Several western countries included & $3(6)$ \\
\hline \multicolumn{2}{|l|}{ Age range } \\
\hline $0-5$ years & $11(22)$ \\
\hline $6-12$ years & $13(27)$ \\
\hline $13-15$ years & $23(47)$ \\
\hline $0-15$ years $^{\mathrm{a}}$ & $2(4)$ \\
\hline \multicolumn{2}{|l|}{ Study design } \\
\hline RCT study & $3(6)$ \\
\hline Quasi-experimental study & $2(4)$ \\
\hline Prospective cohort study & $15(31)$ \\
\hline Cross-sectional study & $29(59)$ \\
\hline \multicolumn{2}{|l|}{ Electronic media devices ${ }^{b}$} \\
\hline Television & $18(37)$ \\
\hline Video game & $12(25)$ \\
\hline Mobile phone/smartphone & $13(27)$ \\
\hline Computer/internet & $14(29)$ \\
\hline Touchscreen/tablet & $3(6)$ \\
\hline Social media & $7(14)$ \\
\hline Total screen time & $43(21)$ \\
\hline Electronic media devices in the bedroom & $5(10)$ \\
\hline Media content (e.g., violent content) & $1(2)$ \\
\hline \multicolumn{2}{|l|}{ Sleep outcome ${ }^{c}$} \\
\hline Delayed bedtime/sleep onset latency & $22(45)$ \\
\hline Poor sleep quality & $19(29)$ \\
\hline Short sleep duration & $36(73)$ \\
\hline Daytime tiredness & $15(31)$ \\
\hline \multicolumn{2}{|c|}{$\begin{array}{l}\text { aWe have predefined three age group categories. If a study included data on } \\
\text { ages overlapping the defined age categories, we used the mean age to } \\
\text { allocate the study to an age category. A few studies }(n=2) \text { included a large } \\
\text { age span } \\
{ }^{b} \text { As some articles examine several electronic media devices within the same } \\
\text { study, this column does not add up to } 100 \% \\
{ }^{C} \text { As some articles examine several sleep outcomes within the same study, this } \\
\text { column does not add up to } 100 \%\end{array}$} \\
\hline
\end{tabular}

later bedtime [18]. Two studies found that electronic media in the bedroom, including mobile phones, televisions, and computers, were associated with later bedtimes $[18,37]$. One of the studies, however, only found a significant association among boys, not girls [37]. One study did not find that electronic media devices in the bedroom was associated with sleep latency and trouble falling asleep [33].

\section{Sleep quality}

Six of eight studies found a positive association between the use of electronic media, including total screen time and bedtime use of television and mobile phone, and night-time awakenings/sleep disturbances [15, 33, 35, 53] or poor sleep quality [20,36]. For example, Mireku et al. (2019) found that using mobile phone or watching television in the dark was associated with restless sleep, waking up at night, and waking early in the morning [36].

\section{Sleep duration}

A total of 15 studies were identified, examining the association between electronic media use and sleep duration among 6-12-year-olds [15-21, 28, 30, 33-37, 53]. The studies found use of mobile phone [17, 33, 35, 36], social media [33], and computer or television [17, 18, 21, 30, $33,36,37]$ associated with short sleep duration. Six studies examined the association between electronic media in the bedroom and sleep duration $[17,18,28,33,34$, 37]. Among these studies, three found an association. Chahal et al. (2012) showed a dose-response association, where children who had access to more electronic media in their bedroom slept less [17]. Falbe et al. (2015) found that children who slept close to a small screen (e.g., mobile phone) reported $21 \mathrm{~min}$ less sleep compared to children who did not [18].

\section{Daytime tiredness}

Two studies examined mobile phone use and daytime tiredness [35, 53]. Redmayne et al. (2013) found that children disturbed by their mobile phone at night at least once a week were 3.5 times more likely to experience daytime tiredness than children who were not disturbed by their mobile phone at night [53]. Huss et al. (2015) did not find an association [35].

\section{Electronic media use among children aged 13-15}

Table 4 summarizes the results of the 24 studies [15, $22-26,38-51,54,55,58,59]$ included in the age range $13-15$.

\section{Bedtime and sleep onset}

Eleven studies investigated the relationship between electronic media use and delayed bedtime and sleep onset $[23,24,40,43,45,46,48-51,59]$. Nine of these studies showed a positive association $[23,40,43,45,46,48$, 49, 51, 59]. High electronic media use was associated with problems falling asleep/later sleep onset [23, 40, 45, 46, 51, 59], delayed bedtime [43, 46, 49] and bedtime problems [45] The study by Poulain et al. (2019) showed a positive association for high use of computer or Internet (3-4h/day or more) and more bedtime problems at 12-month follow-up, while no association was found for television viewing or mobile phone use [45]. Two studies 
Table 2 Summary of studies and their findings on the relationship between electronic media devices and sleep outcomes among 0-5-year-olds

\begin{tabular}{|c|c|c|c|c|c|c|c|}
\hline \multirow{2}{*}{$\begin{array}{l}\text { Electronic media } \\
\text { device }\end{array}$} & \multirow{2}{*}{$\begin{array}{l}\text { Measured at } \\
\text { bedtime }\end{array}$} & \multicolumn{4}{|l|}{ Sleep outcomes } & \multirow[t]{2}{*}{ Study design } & \multirow{2}{*}{$\begin{array}{l}\text { Quality } \\
\text { Assessmen }\end{array}$} \\
\hline & & $\begin{array}{l}\text { Delayed bedtime } \\
\text { or sleep onset } \\
\text { latency }\end{array}$ & $\begin{array}{l}\text { Poor sleep } \\
\text { quality }\end{array}$ & $\begin{array}{l}\text { Short sleep } \\
\text { duration }\end{array}$ & $\begin{array}{l}\text { Daytime sleep } \\
\text { duration/ Daytime } \\
\text { tiredness }\end{array}$ & & \\
\hline \multicolumn{8}{|l|}{ Television } \\
\hline \multirow[t]{2}{*}{ Beyens 2019} & No & + & & $+/ 0$ & + & Cross-sectional & Moderate \\
\hline & Yes & + & & $+/ 0$ & 0 & & \\
\hline Cespedes 2014 & No & & & + & & Cohort study & Moderate \\
\hline Cheung 2017 & No & 0 & 0 & 0 & + & Cross-sectional & Moderate \\
\hline Marinelli 2014 & No & & & + & & Cohort study & Moderate \\
\hline McDonald 2014 & Yes & & & + & & Cross-sectional & Moderate \\
\hline \multirow[t]{2}{*}{ Moorman 2019} & No & 0 & & + & + & Cross-sectional & Moderate \\
\hline & Yes & + & & + & 0 & & \\
\hline Plancoulaine 2018 & No & & & + & & Cohort study & Moderate \\
\hline \multicolumn{8}{|c|}{ Video game (console) } \\
\hline \multirow[t]{2}{*}{ Beyens 2019} & No & 0 & & 0 & 0 & Cross-sectional & Moderate \\
\hline & Yes & 0 & & 0 & 0 & & \\
\hline \multirow[t]{2}{*}{ Moorman 2019} & No & + & & 0 & 0 & Cross-sectional & Moderate \\
\hline & Yes & + & & 0 & & & \\
\hline \multirow[t]{2}{*}{ Nathanson 2018} & No & 0 & & 0 & 0 & Cross-sectional & Moderate \\
\hline & Yes & 0 & & 0 & 0 & & \\
\hline \multicolumn{8}{|l|}{ Mobile phone } \\
\hline \multirow[t]{2}{*}{ Beyens 2019} & No & 0 & & 0 & $+/ 0$ & Cross-sectional & Moderate \\
\hline & Yes & 0 & & 0 & + & & \\
\hline \multirow[t]{2}{*}{ Nathanson 2018} & No & 0 & & 0 & 0 & Cross-sectional & Moderate \\
\hline & Yes & + & & 0 & 0 & & \\
\hline \multicolumn{8}{|l|}{ Touchscreen/tablet } \\
\hline \multirow[t]{2}{*}{ Beyens 2019} & No & + & & $+/ 0$ & + & Cross-sectional & Moderate \\
\hline & Yes & + & & $+/ 0$ & $+/ 0$ & & \\
\hline Cheung 2017 & No & + & 0 & + & 0 & Cross-sectional & Moderate \\
\hline \multirow[t]{2}{*}{ Nathanson 2018} & No & + & & + & 0 & Cross-sectional & Moderate \\
\hline & Yes & + & & + & 0 & & \\
\hline \multicolumn{8}{|l|}{ Computer/internet } \\
\hline \multirow[t]{2}{*}{ Beyens 2019} & No & 0 & & 0 & 0 & Cross-sectional & Moderate \\
\hline & Yes & 0 & & 0 & 0 & & \\
\hline \multirow[t]{2}{*}{ Moorman 2019} & No & 0 & & $+/ 0$ & 0 & Cross-sectional & Moderate \\
\hline & Yes & 0 & & 0 & 0 & & \\
\hline \multirow[t]{2}{*}{ Nathanson 2018} & No & 0 & & 0 & 0 & Cross-sectional & Moderate \\
\hline & Yes & 0 & & 0 & 0 & & \\
\hline \multicolumn{8}{|l|}{ Total screen time } \\
\hline Parent 2016 & No & & + & + & & Cross-sectional & Moderate \\
\hline Ribner 2019 & No & & & + & 0 & Cross-sectional & Moderate \\
\hline Xu 2016 & No & + & - & + & & Cohort study & Moderate \\
\hline Zhang 2018 & No & & 0 & 0 & & Cross-sectional & Moderate \\
\hline
\end{tabular}


Table 2 Summary of studies and their findings on the relationship between electronic media devices and sleep outcomes among 0-5-year-olds (Continued)

\begin{tabular}{|c|c|c|c|c|c|c|c|}
\hline \multirow{2}{*}{$\begin{array}{l}\text { Electronic media } \\
\text { device }\end{array}$} & \multirow{2}{*}{$\begin{array}{l}\text { Measured at } \\
\text { bedtime }\end{array}$} & \multicolumn{4}{|l|}{ Sleep outcomes } & \multirow[t]{2}{*}{ Study design } & \multirow{2}{*}{$\begin{array}{l}\text { Quality } \\
\text { Assessmen }\end{array}$} \\
\hline & & $\begin{array}{l}\text { Delayed bedtime } \\
\text { or sleep onset } \\
\text { latency }\end{array}$ & $\begin{array}{l}\text { Poor sleep } \\
\text { quality }\end{array}$ & $\begin{array}{l}\text { Short sleep } \\
\text { duration }\end{array}$ & $\begin{array}{l}\text { Daytime sleep } \\
\text { duration/ Daytime } \\
\text { tiredness }\end{array}$ & & \\
\hline \multicolumn{8}{|c|}{ Electronic media devices in the bedroom } \\
\hline Cespedes 2014 & & & & $+/ 0$ & & Cohort study & Moderate \\
\hline Moorman 2019 & & + & & 0 & 0 & Cross-sectional & Moderate \\
\hline \multicolumn{8}{|l|}{ Media content } \\
\hline Garrison 2012 & No & & + & & & $\mathrm{RCT}$ & Moderate \\
\hline
\end{tabular}

Note: 1st author and year. (+) Significant positive association; (0) No association; (-) Significant negative association; (+/0) refers to both a positive association and no association in different subgroups

found an association between social media use and delayed sleep onset $[46,48]$. Van der Schuur et al. (2019) found that social media stress was longitudinally related to sleep onset latency among girls, but not boys [48] Scott et al. (2019) found a dose-response relationship between social media use and late sleep onset, where a higher use of social media was associated with higher odds of late sleep onset [46].

\section{Sleep quality}

Nine studies assessed the association between electronic media use and sleep quality, including restless sleep, night-time awakenings, and insomnia complaints [15, $40,41,44-46,50,54,55]$ and seven of these studies found a positive association [15, 40, 41, 44, 46, 54, 55]. Three of these studies examined social media use [46, $54,55]$. There were indications that a large amount of time on social media or problematic use of social media had an impact on sleep quality $[46,54,55]$. Problematic use of social media was measured by whether the adolescents preferred spending time on social media rather than engaging in social activities or used social media to feel good about themselves.

\section{Sleep duration}

The relationship between electronic media use and sleep duration was examined in 13 studies [15, 22-26, 38, 39, 42, 50, 51, 58, 59]. Mazzer et al. (2018), who examined eighth- and ninth-grade students over a year, found that of electronic media use was associated with short sleep duration [42]. Regarding different types of electronic media, it appears that computers [22, 24, 38, 58], mobile phones [22, 24, 38], and video games [22, 38] affected sleep duration. Brunetti et al. (2016), for example, found that computer use doubled the odds of a short night's sleep, while talking on a mobile phone tripled the odds of a short night's sleep [22]. In contrast, Tavernier et al. (2017) found that talking on the phone increased the sleep duration, whereas texting reduced the sleep duration. In this study, social media use was not related to sleep duration, whereas Twenge et al. (2017) found an association between social media and short sleep duration. Two studies investigated the presence of electronic media in the bedroom and sleep duration. There were indications that a computer, but not a television or gaming console, in the bedroom negatively affected sleep duration $[23,39]$.

\section{Daytime tiredness}

Eight studies examined the association between electronic media use and daytime tiredness, and found mixed results $[22,23,40,45,47-50]$. Poulain et al. (2019) showed that high computer or Internet use, but not television and mobile phone use, resulted in more daytime tiredness [45]. Brunetti et al. (2016) found that computer use and time spent talking on the mobile phone were associated with more daytime sleepiness while no associations were found for videogame time and television use [22]. One study that examined social media showed that using social media was not in itself associated with daytime tiredness, but adverse emotional reactions arising from social media (i.e. social media stress) was related to daytime tiredness among girls [48].

\section{Discussion}

This systematic review summarizes results from 49 epidemiological studies on associations between electronic media use and sleep in 0-15-year-old children and adolescents. Across age groups, we found consistent evidence that media use was associated with short sleep duration. The evidence for a relationship between electronic media use and other sleep outcomes was less strong.

For the youngest children (i.e. preschool children), television watching, and tablet device use were associated with difficulties in falling asleep and less sleep duration. Moreover, heavier television use was associated with increased daytime napping, which suggests poorer 
Table 3 Summary of studies and their findings on the relationship between electronic media devices and sleep outcomes among 6-12-year-olds

\begin{tabular}{|c|c|c|c|c|c|c|c|}
\hline \multirow{2}{*}{$\begin{array}{l}\text { Electronic media } \\
\text { device }\end{array}$} & \multirow{2}{*}{$\begin{array}{l}\text { Measured at } \\
\text { bedtime }\end{array}$} & \multicolumn{4}{|l|}{ Sleep outcomes } & \multirow[t]{2}{*}{ Study design } & \multirow{2}{*}{$\begin{array}{l}\text { Quality } \\
\text { Assessmen }\end{array}$} \\
\hline & & $\begin{array}{l}\text { Delayed bedtime or } \\
\text { sleep onset latency }\end{array}$ & $\begin{array}{l}\text { Poor sleep } \\
\text { quality }\end{array}$ & $\begin{array}{l}\text { Short sleep } \\
\text { duration }\end{array}$ & $\begin{array}{l}\text { Daytime } \\
\text { tiredness }\end{array}$ & & \\
\hline \multicolumn{8}{|l|}{ Television } \\
\hline Arora 2014 & Yes & 0 & + & + & & Cross-sectional & Moderate \\
\hline Chahal 2012 & Yes & & & + & & Cohort study & Moderate \\
\hline Falbe 2014 & No & + & & + & & Cross-sectional & Moderate \\
\hline Marinelli 2014 & No & & & + & & Cohort study & Moderate \\
\hline Mireku 2019 & Yes & + & + & + & & Cross-sectional & Moderate \\
\hline Nuutinen 2013 & No & + & & + & & Cohort study & Moderate \\
\hline Yland 2015 & No & & & + & & Cross-sectional & Moderate \\
\hline \multicolumn{8}{|c|}{ Video game (console) } \\
\hline Arora 2014 & Yes & + & + & + & & Cross-sectional & Moderate \\
\hline Chahal 2012 & Yes & & & + & & Cohort study & Moderate \\
\hline Falbe 2014 & No & + & & + & & Cross-sectional & Moderate \\
\hline Yland 2015 & No & & & 0 & & Cross-sectional & Moderate \\
\hline \multicolumn{8}{|l|}{ Mobile phone } \\
\hline Arora 2014 & Yes & $+/ 0$ & + & + & & Cross-sectional & Moderate \\
\hline Chahal 2012 & Yes & & & + & & Cohort study & Moderate \\
\hline Huss 2015 & No & + & + & + & 0 & Cohort study & Moderate \\
\hline Mireku 2019 & Yes & + & + & + & & Cross-sectional & Moderate \\
\hline Redmayne 2013 & No & 0 & 0 & 0 & + & Cross-sectional & Moderate \\
\hline \multicolumn{8}{|l|}{ Computer } \\
\hline Arora 2014 & Yes & $+/ 0$ & + & + & & Cross-sectional & Moderate \\
\hline Chahal 2012 & Yes & & & + & & Cohort study & Moderate \\
\hline Nuutinen 2013 & No & + & & + & & Cohort study & Moderate \\
\hline Yland 2015 & No & & & $+/ 0$ & & Cross-sectional & Moderate \\
\hline \multicolumn{8}{|c|}{ Internet/social media } \\
\hline Arora 2014 & Yes & + & 0 & + & & Cross-sectional & Moderate \\
\hline \multicolumn{8}{|l|}{ Total screen time } \\
\hline Barlett 2011 & No & & & + & & Cohort study & Moderate \\
\hline Brambilla 2017 & Yes & & & + & & Cross-sectional & Moderate \\
\hline Gentile 2014 & No & & & + & & Cohort study & Moderate \\
\hline Greever 2017 & No & & + & 0 & & Cross-sectional & Moderate \\
\hline Mireku 2019 & Yes & + & & + & & Cross-sectional & Moderate \\
\hline Parent 2016 & No & & + & + & & Cross-sectional & Moderate \\
\hline \multicolumn{8}{|c|}{ Electronic media devices in the bedroom } \\
\hline Arora 2014 & & 0 & 0 & + & & Cross-sectional & Moderate \\
\hline Brambilla 2017 & & & & 0 & & Cross-sectional & Moderate \\
\hline Chahal 2012 & & & & + & & Cohort study & Moderate \\
\hline Falbe 2014 & & + & & + & & Cross-sectional & Moderate \\
\hline Mindell 2016 & & & & 0 & & $\mathrm{RCT}$ & Moderate \\
\hline Nuutinen 2013 & & + & & $+/ 0$ & & Cohort study & Moderate \\
\hline
\end{tabular}


Table 4 Summary of studies and their findings on the relationship between electronic media devices and sleep outcomes among 13-15-year-olds

\begin{tabular}{llllll}
\hline $\begin{array}{l}\text { Electronic media } \\
\text { device }\end{array}$ & $\begin{array}{l}\text { Measured } \\
\text { at } \\
\text { bedtime }\end{array}$ & \multicolumn{2}{l|}{ Sleep outcomes } & Study design & $\begin{array}{l}\text { Quality } \\
\text { Assessment }\end{array}$ \\
\cline { 2 - 4 } & $\begin{array}{l}\text { Delayed } \\
\text { bedtime or } \\
\text { sleep onset } \\
\text { latency }\end{array}$ & $\begin{array}{l}\text { Poor sleep } \\
\text { quality }\end{array}$ & $\begin{array}{l}\text { Short sleep } \\
\text { duration }\end{array}$ & $\begin{array}{l}\text { Daytime } \\
\text { tiredness }\end{array}$ & \\
\hline
\end{tabular}

\section{Television}

Arora 2013

Brunetti 2016

Lange 2015

Poulain 2019

Tavenier 2017

Twenge 2017

Video game (console)

Arora 2013

Brunetti 2016

Lange 2015

Tavernier 2017

Wallenius 2009

$\begin{array}{ll}\text { Yes } & \\ \text { No } & \\ \text { No } & \\ \text { No } & 0 \\ \text { No } & 0 \\ \text { No } & \end{array}$

$\begin{array}{ll} & \\ & + \\ 0 & \\ 0 & \\ & \\ & \\ & +10\end{array}$

0

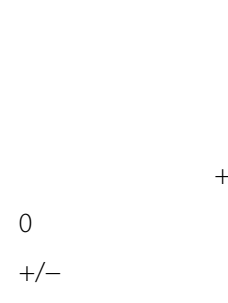

Mobile phone/smartphone

Arora 2013
Brunetti 2016
Foerster 2019
Lange 2015
Poulain 2019
Tavernier 2017
Computer/internet

Computer/internet

Arora 2013
Brunetti 2016
Lange 2015
Nuutinen 2014
Poulain 2019
Tavernier 2017
Twenge 2017
Social media

\section{Social media}

Scott 2019

Tavernier 2017

Twenge 2017

Van der Schuur 2019

Vernon 2015

Vernon 2017

\section{Total screen time}

Bickham 2018

Calamaro 2009

Das_Friebel 2018

Foerster 2019
Yes

No

No

No

No

Yes

No

No

No

No

No

Yes

No

No

No

No

No

No

No

No

No

No

No

No

No

Yes

Yes

No

$\begin{array}{ll}\text { Cross-sectional } & \text { Moderate } \\ \text { Cross-sectional } & \text { Moderate } \\ \text { Cross-sectional } & \text { Moderate } \\ \text { Cohort study } & \text { High } \\ \text { Cohort study } & \text { High } \\ \text { Cross-sectional } & \text { Moderate } \\ & \\ \text { Cross-sectional } & \text { Moderate } \\ \text { Cross-sectional } & \text { Moderate } \\ \text { Cross-sectional } & \text { Moderate } \\ \text { Cohort study } & \text { High } \\ \text { Cross-sectional } & \text { Moderate }\end{array}$

Cross-sectional

Moderate

Cross-sectional

Moderate

Cohort study

Moderate

Cross-sectional

Moderate

Cohort study

High

Cohort study

High

Cross-sectional

Moderate

Cross-sectional

Moderate

Cross-sectional

Moderate

Cross-sectional

Moderate

Cohort study

High

Cohort study

High

Cross-sectional

Moderate

Cross-sectional

Moderate

Cohort study

High

Cross-sectional

Moderate

$+/ 0$

Cohort study

Moderate

Cross-sectional

Moderate

Cohort study

Moderate

$\begin{array}{ll}\text { Quasi-experimental } & \text { High } \\ \text { Cross-sectional } & \text { Moderate } \\ \text { RCT } & \text { High } \\ \text { Cohort study } & \text { Moderate }\end{array}$


Table 4 Summary of studies and their findings on the relationship between electronic media devices and sleep outcomes among 13-15-year-olds (Continued)

\begin{tabular}{|c|c|c|c|c|c|c|c|}
\hline \multirow{2}{*}{$\begin{array}{l}\text { Electronic media } \\
\text { device }\end{array}$} & \multirow{2}{*}{$\begin{array}{l}\text { Measured } \\
\text { at } \\
\text { bedtime }\end{array}$} & \multicolumn{4}{|c|}{ Sleep outcomes } & \multirow[t]{2}{*}{ Study design } & \multirow{2}{*}{$\begin{array}{l}\text { Quality } \\
\text { Assessment }\end{array}$} \\
\hline & & $\begin{array}{l}\text { Delayed } \\
\text { bedtime or } \\
\text { sleep onset } \\
\text { latency }\end{array}$ & $\begin{array}{l}\text { Poor sleep } \\
\text { quality }\end{array}$ & $\begin{array}{l}\text { Short sleep } \\
\text { duration }\end{array}$ & $\begin{array}{l}\text { Daytime } \\
\text { tiredness }\end{array}$ & & \\
\hline Lange 2015 & No & & $+/ 0$ & & & Cross-sectional & Moderate \\
\hline Mazzer 2018 & No & & & + & & Cohort study & Moderate \\
\hline Ogunleye 2015 & No & + & & & & Cross-sectional & Moderate \\
\hline Ononogbu 2014 & No & & + & & & Cross-sectional & Moderate \\
\hline Parent 2016 & No & & + & 0 & & Cross-sectional & Moderate \\
\hline Perrault 2019 & Yes & + & & + & & Quasi-experimental & Moderate \\
\hline Twenge 2017 & No & & & + & & Cross-sectional & Moderate \\
\hline Van der Schuur 2018 & No & & 0 & & 0 & Cohort study & Moderate \\
\hline Vandendriessche 2019 & No & + & & + & & Cross-sectional & Moderate \\
\hline \multicolumn{8}{|c|}{ Electronic media devices in the bedroom } \\
\hline Calamaro 2009 & & & & 0 & & Cross-sectional & Moderate \\
\hline Continente 2016 & & & & $+/ 0$ & & Cross-sectional & Moderate \\
\hline
\end{tabular}

Note: 1st author and year. (+) Significant positive association; (0) No association; (-) Significant negative association; (+/0) refers to both a positive association and no association in different subgroups; (+/-) refers to both a positive association and a negative association in different subgroups

sleep consolidation and less mature sleep patterns [60]. There was no or insufficient evidence for an association of video game, mobile phone, computer, and the presence of an electronic media device in the bedroom with poor sleep outcomes among 0 -5-year-olds.

For 6-12-year-old children, use of electronic media (television, video game console, mobile phone, computer, screen time) in general and at bedtime and their presence in the bedroom was associated with later bedtimes and shorter sleep duration. Additionally, we found evidence for an association of bedtime television and mobile phone use as well as total screen time with sleep disturbances and awakening at night. Similar results have been reported in a previous systematic review examining the association between portable screenbased media device access or use in the bedroom and less sleep [61]. Our results support the hypothesis that evening exposure to bright light from screens may disturb the sleep-wake cycle and suppresses the melatonin production $[7,62]$. Other mechanisms through which media use may interfere with sleep onset and sleep problems are time replacement (i.e. time spent on the screens at night displaces time spent sleeping) or the psychological stimulation from the media content [63].

For 13-15-year-olds, there was evidence for a positive association of total screen time and use of computer and mobile phone with less sleep. Moreover, the included studies indicated that screen time was associated with problems falling asleep, and social media use was associated with poor sleep quality. Television watching was least likely to be associated with poor sleep outcomes. Thus, for this age group our study supports that more interactive forms of electronic media with increases in physiological arousal [64] may have greater impact on sleep than more passive forms $[7,65]$. Our results are in line with a systematic review [66] including an older age group (15-24-year-olds) that suggests that adolescents often use electronic devices for social media, and this may explain the relationship between use of electronic media device and poor sleep.

Five of the included studies investigated subgroup effect or effect modification, i.e. whether an observed association differed depending on characteristics of the study sample. Four studies performed genderspecific analyses [39, 40, 48, 49] and one study examined ethnicity [12]. The studies found that the association between electronic media use and sleep may depend on gender and ethnicity. For example, a study from US found that bedroom TV was associated with less sleep for ethnic minority children but not among white non-Hispanic children [12]. Future studies of properly powered subgroup analysis should further investigate whether the association between media use and sleep in children and adolescent is differentially impacted by factors such as age and ethnicity [67]. Moreover, how does parenting style, values, and socioeconomic status of the family contribute to the impact of electronic media on sleep. Improvements in sociocultural and contextual understanding would 
elucidate the association between media use and sleep in childhood and adolescence [68].

\section{Limitations and future directions}

We noted several limitations in the studies included in this review. Firstly, most of the study designs were crosssectional which precluded causal inferences and limit the conclusions of this review because it is not possible to uncover the direction of relationships between electronic media and sleep. Some children and adolescents may experience bedtime procrastination (i.e. going to bed later than intended despite the absence of external reasons) [69] and use electronic media as an activity before sleep. Others may use electronic media to help them go to sleep [70] or because of tiredness [42]. Such reciprocal associations are confirmed by some of the included studies [42, 45]. Prospective studies with measurements at multiple time points are needed to identify how and when use of electronic media impact on sleep in childhood and youth. Secondly, both self-reported and parent-reported data may be subject to uncertainty. For example, adolescents tend to over-report their sleep duration compared with objective measurements such as actigraphy or diary methods [71], and parents tend to report better sleep for adolescents compared with both self-reported and objective measurements [72]. Thirdly, the included studies were measuring media use and sleep outcomes differently (e.g., overall screen time exposure versus bedtime use). Due to the substantial heterogeneity in measurements of media and sleep as well as in effect size measure, it was difficult to summarize the results, estimate the magnitude of the associations and provide clear conclusions. Nonetheless, we have rigorously outlined the associations between electronic media and each sleep outcome which enables comprehensive results; still, there were several insufficiencies. The studies among 6-12-year-olds lacked measurements of sleepiness during daytime; a factor that may have serious consequences on schoolwork and leisure activities. In the age group of 13-15-year-olds, there was a lack of studies measuring the electronic media use at bedtime or during the night. This is an important area to examine because parental monitoring and parent-set bedtimes decline significantly from early to late years of adolescence [73] which might imply an increase in the use of electronic media devices at bedtime.

This review was limited to include studies published in English and study populations from Western countries. The latter make us able to generalize to Western countries but limits the generalizability of our findings to non-Western countries. The reason for the inclusion criteria was that traditions, values, conditions and environments for sleep practices and attitudes may differ between Western and non-Western countries [74], inhibiting results to inform policy and practice.

Despite these limitations, we note several strengths of this study in addressing the association between electronic media device use and sleep in children and adolescents. First, the studies represented a relatively short period of time which ensured that the definition of electronic media devices remained stable. Secondly, we excluded studies of low quality. Thirdly, we included a broad age group; children and adolescents from 0 to 15 . This broadens our understanding of how the use of electronic media devices may impact sleep in different age groups in a childhood development perspective. However, comprehensive equity reviews on e.g. socioeconomic background in the study population and mechanisms would provide a more thorough understanding of the associations. Only a few qualitative studies were identified. This leaves a large gap in understanding the complexities of electronic media device use and sleep relationship in children and adolescents.

\section{Implications for policy and practice}

At the policy level, information and more public awareness could be promoted about the potential negative impact on children's sleep of electronic media, if used excessively and immediately before bedtime. In general, this could include renewed awareness and promotion of appropriate sleep hygiene, but also more attention to the potential adverse effects of the seemingly unavoidable increase in the use of electronic media in the everyday life. At the practice level, professionals and caretakers of children and adolescents should have a heightened awareness on sleep and encourage bedtime routines including calming activities without use of electronic media and remove all electronic media from the bedroom [75]. Given the strong attraction of electronic media on most children, interventions should include both structural measures to guide children's electronic media habits and individual measures focusing on e.g. information about the potential impact of electronic media devices and how to develop healthy media habits.

\section{Conclusions}

This systematic review of 49 studies found consistent evidence that use of electronic devices is associated with shorter sleep duration in children and adolescents. The association between electronic media use and other sleep outcomes was more inconclusive. Moreover, the evidence for association between electronic media and sleep was stronger for $6-15$-years-olds than $0-5$-yearolds. 


\section{Abbreviations}

$\mathrm{N}$ : Number; RCT: Randomized controlled trial

\section{Supplementary Information}

The online version contains supplementary material available at https://doi. org/10.1186/s12889-021-11640-9.

\section{Additional file 1: Table S1: Search terms and syntax. Table S2}

Characteristics and detailed results of included studies among 0-5-year-

old children. Table S3: Characteristics and detailed results of included

studies among 6-12-year-old children. Table S4: Characteristics and

detailed results of included studies among 13-15-year-old children.

Table S5: References on excluded studies.

\section{Acknowledgements}

We would like to thank all the authors of the primary studies included in this systematic review.

\section{Authors' contributions}

Affiliation: National Institute of Public Health, University of Southern Denmark, Copenhagen, Denmark.

Contributions: $L L$ and SA designed the study. $L L$ and ISN conducted the database searches and extracted data. LL, ISN and SA did the initial analysis and DD supervised data analysis. LL and SA wrote the first draft, and ISN and DD critically revised the first draft. All authors have read, reviewed, and approved the final manuscript.

\section{Funding}

No specific funding was received for this article; however, we gratefully acknowledge the support of the The Danish Health Authority. The content is solely the responsibility of the authors. The Danish Health Authority was not involved in designing the study, collecting, analyzing or interpreting data, in writing manuscript, or in the decision to submit the manuscript for publication

\section{Availability of data and materials}

All data generated or analyzed during this study are included in this article (and its supplementary files).

\section{Declarations}

\section{Ethics approval and consent to participate}

Ethics approval and consent to participate was not required for this review.

\section{Consent for publication}

Not applicable.

\section{Competing interests}

The authors declare no conflict of interest.

Received: 5 January 2021 Accepted: 18 August 2021

Published online: 30 September 2021

\section{References}

1. Dewald JF, Meijer AM, Oort FJ, Kerkhof GA, Bogels SM. The influence of sleep quality, sleep duration and sleepiness on school performance in children and adolescents: a meta-analytic review. Sleep Med Rev. 2010;14(3): 179-89. https://doi.org/10.1016/j.smrv.2009.10.004.

2. Owens J. Adolescent sleep working group, committee on adolescence. Insufficient sleep in adolescents and young adults: an update on causes and consequences. Pediatrics. 2014;134(3):e921-32. https://doi.org/10.1542/ peds.2014-1696.

3. Bagley EJ, Kelly RJ, Buckhalt JA, El-Sheikh M. What keeps low-SES children from sleeping well: the role of presleep worries and sleep environment. Sleep Med. 2015;16(4):496-502

4. Reid Chassiakos Y, Radesky J, Christakis D, et al. AAP COUNCIL ON COMMUNICATIONS AND MEDIA. Children and Adolescents and Digita Media. Pediatrics. 2016;138(5):e20162593.
5. Vanden AM. Mobile youth culture: a conceptual development. Mobile Media Commun. 2016:4(1):85-101. https://doi.org/10.1177/20501579156014 55.

6. Hale L, Guan S. Screen time and sleep among school-aged children and adolescents: a systematic literature review. Sleep Med Rev. 2015;21:50-8. https://doi.org/10.1016/j.smrv.2014.07.007.

7. Cain N, Gradisar M. Electronic media use and sleep in school-aged children and adolescents: a review. Sleep Med. 2010;11(8):735-42. https://doi.org/1 0.1016/j.sleep.2010.02.006.

8. Carter B, Rees P, Hale L, Bhattacharjee D, Paradkar MS. Association between portable screen-based media device access or use and sleep outcomes a systematic review and meta-analysis. JAMA Pediatr. 2016;170(12):1202-8. https://doi.org/10.1001/jamapediatrics.2016.2341.

9. Chaput J-P, Gray CE, Poitras VJ, Carson V, Gruber R, Birken CS, et al. Systematic review of the relationships between sleep duration and health indicators in the early years (0-4 years). BMC Public Health. 2017;17:91-107.

10. McMaster University. Effective public health practice quality assessment tool for quantitative studies: McMaster University School of Nursing; 2008.

11. Beyens I, Nathanson Al. Electronic media use and sleep among preschoolers: evidence for time-shifted and less consolidated sleep. Health Commun. 2019;34(5):537-44. https://doi.org/10.1080/10410236.2017.14221 02.

12. Cespedes EM, Gillman MW, Kleinman K, Rifas-Shiman SL, Redline S, Taveras EM. Television viewing, bedroom television, and sleep duration from infancy to mid-childhood. Pediatrics. 2014;133(5):e1163-e71. https://doi.org/10.1542/ peds.2013-3998.

13. Moorman JD, Harrison K. Beyond access and exposure: implications of sneaky media use for Preschoolers' sleep behavior. Health Commun. 2019; 34(5):529-36. https://doi.org/10.1080/10410236.2017.1422103.

14. Nathanson Al, Beyens I. The relation between use of Mobile electronic devices and bedtime resistance, sleep duration, and daytime sleepiness among preschoolers. Behav Sleep Med. 2018;16(2):202-19. https://doi.org/1 0.1080/15402002.2016.1188389

15. Parent J, Sanders W, Forehand R. Youth screen time and behavioral health problems: the role of sleep duration and disturbances. J Dev Behav Pediatrics. 2016;37(4):277-84. https://doi.org/10.1097/DBP.00000000000002 72

16. Barlett ND, Gentile DA, Barlett CP, Eisenmann JC, Walsh DA. Sleep as a mediator of screen time effects on US children's health outcomes: A prospective study. J Children Media. 2012;6(1):37-50. https://doi.org/10.1 080/17482798.2011.633404

17. Chahal H, Fung C, Kuhle S, Veugelers PJ. Availability and night-time use of electronic entertainment and communication devices are associated with short sleep duration and obesity among Canadian children. Pediatric Obesity. 2012;8(1):42-51. https://doi.org/10.1111/j.2047-6310.2012.00085.X.

18. Falbe J, Davison KK, Franckle RL, Ganter C, Gortmaker SG, Smith L, et al. Sleep duration, restfulness, and screens in the sleep environment. Pediatrics. 2015;135(2):e367-e75. https://doi.org/10.1542/peds.2014-2306.

19. Gentile DA, Reimer RA, Nathanson Al, Walsh DA, Eisenmann JC. Protective effects of parental monitoring of children's media use a prospective study JAMA Pediatr. 2014;168(5):479-84. https://doi.org/10.1001/jamapediatrics.2 014.146.

20. Greever CJ, Ahmadi M, Sirard J, Alhassan S. Associations among physical activity, screen time, and sleep in low socioeconomic status urban girls. Prev Med Rep. 2017;5:275-8. https://doi.org/10.1016/j. pmedr.2017.01.014

21. Yland J, Guan S, Emanuele E, Hale L. Interactive vs passive screen time and nighttime sleep duration among school-aged children. Sleep Health. 2015; 1(3):191-6. https://doi.org/10.1016/j.sleh.2015.06.007.

22. Brunetti VC, O'Loughlin EK, O'Loughlin J, Constantin E, Pigeon E. Screen and nonscreen sedentary behavior and sleep in adolescents. Sleep Health. 2016; 2(4):335-40. https://doi.org/10.1016/j.sleh.2016.09.004.

23. Calamaro CJ, Mason TA, Ratcliffe S. Adolescents living the $24 / 7$ lifestyle: Effects of caffeine and technology on sleep duration and daytime functioning. Sleep. 2009;S:A67-A8.

24. Tavernier R, Heissel JA, Sladek MR, Grant KE, Adam EK. Adolescents' technology and face-to-face time use predict objective sleep outcomes. Sleep Health. 2017;3(4):276-83. https://doi.org/10.1016/j.sleh.2017.04.005

25. Twenge JM, Krizan Z, Hisler G. Decreases in self-reported sleep duration among U.S. adolescents 2009-2015 and association with new media screen time. Sleep Med. 2017;39:47-53. https://doi.org/10.1016/j.sleep.2017.08.013. 
26. Bickham DS, Hswen $Y$, Slaby RG, Rich M. A preliminary evaluation of a school-based media education and reduction intervention. J Prim Prev. 2018;39(3):229-45. https://doi.org/10.1007/s10935-018-0510-2.

27. Garrison MM, Christakis DA. The impact of a healthy media use intervention on sleep in preschool children. Pediatrics. 2012;130(3):492-9. https://doi. org/10.1542/peds.2011-3153.

28. Mindell JA, Sedmak R, Boyle JT, Butler R, Williamson AA. Sleep well!: a pilot study of an education campaign to improve sleep of socioeconomically disadvantaged children. J Clin Sleep Med. 2016;12(12):1593-9. https://doi. org/10.5664/jcsm.6338.

29. Cheung CH, Bedford R, Saez De Urabain IR, Karmiloff-Smith A, Smith TJ. Daily touchscreen use in infants and toddlers is associated with reduced sleep and delayed sleep onset. Sci Rep. 2017;7:46104.

30. Marinelli M, Sunyer J, Alvarez-Pedrerol M, Iñiguez C, Torrent M, Vioque J, et al. Hours of television viewing and sleep duration in children: a multicenter birth cohort study. JAMA Pediatr. 2014;168(5):458-64. https:// doi.org/10.1001/jamapediatrics.2013.3861.

31. McDonald L, Wardle J, Llewellyn $\mathrm{CH}$, van Jaarsveld CHM, Fisher A. Predictors of shorter sleep in early childhood. Sleep Med. 2014;15(5):536-40. https:// doi.org/10.1016/j.sleep.2014.01.005.

32. Plancoulaine S, Forhan A, Lioret S, Heude B, Charles M-A, Reynaud E. Night sleep duration trajectories and associated factors among preschool children from the EDEN cohort. Sleep Med. 2018;48:194-201. https://doi.org/10.1016/ j.sleep.2018.03.030.

33. Arora T, Broglia E, Thomas GN, Taheri S. Associations between specific technologies and adolescent sleep quantity, sleep quality, and parasomnias. Sleep Med. 2014;15(2):240-7. https://doi.org/10.1016/j.sleep.2013.08.799.

34. Brambilla P, Giussani M, Pasinato A, Venturelli L, Privitera F, Giudice EM, et al. Sleep habits and pattern in 1-14 years old children and relationship with video devices use and evening and night child activities. Ital J Pediatr. 2017:43:1-11.

35. Huss A, Van Eijsden M, Guxens M, Beekhuizen J, Van Strien R, Kromhout H, et al. Environmental radiofrequency electromagnetic fields exposure at home, mobile and cordless phone use, and sleep problems in 7-year-old children. PLoS One. 2015;10(10):e0139869.

36. Mireku MO, Barker MM, Mutz J, Dumontheil I, Thomas MSC, Roosli M, et al. Night-time screen-based media device use and adolescents' sleep and health-related quality of life. Environ Int. 2019;124:66-78. https://doi.org/10.1 016/j.envint.2018.11.069.

37. Nuutinen T, Ray C, Roos E. Do computer use, TV viewing, and the presence of the media in the bedroom predict school-aged children's sleep habits in a longitudinal study? BMC Public Health. 2013;13(1):684. https://doi.org/1 0.1186/1471-2458-13-684

38. Arora T, Hussain S, Hubert Lam KB, Lily Yao G, Neil Thomas G, Taheri S. Exploring the complex pathways among specific types of technology, selfreported sleep duration and body mass index in UK adolescents. Int J Obes. 2013;37(9):1254-60. https://doi.org/10.1038/ijo.2012.209.

39. Continente X, Pérez A, Espelt A, López MJ. Media devices, family relationships and sleep patterns among adolescents in an urban area. Sleep Med. 2017;32:28-35. https://doi.org/10.1016/j.sleep.2016.04.006.

40. Foerster M, Henneke A, Chetty-Mhlanga S, Roosli M. Impact of Adolescents' screen time and nocturnal Mobile phone-related awakenings on sleep and general health symptoms: a prospective cohort study. Int J Environ Res Public Health. 2019;16(3):12.

41. Lange K, Cohrs S, Skarupke C, Gorke M, Szagun B, Schlack R. Electronic media use and insomnia complaints in German adolescents: gender differences in use patterns and sleep problems. J Neural Transm. 2017; 124(Suppl 1):79-87. https://doi.org/10.1007/s00702-015-1482-5.

42. Mazzer K, Bauducco S, Linton SJ, Boersma K. Longitudinal associations between time spent using technology and sleep duration among adolescents. J Adolesc. 2018;66:112-9. https://doi.org/10.1016/j.a dolescence.2018.05.004.

43. Ogunleye AA, Voss C, Sandercock GR. Delayed bedtime due to screen time in schoolchildren: importance of area deprivation. Pediatr Int. 2015;57(1): 137-42. https://doi.org/10.1111/ped.12447.

44. Ononogbu S, Wallenius M, Punamäki RL, Saarni L, Lindholm H, Nygård $\mathrm{CH}$. Association between Information and communication technology usage and the quality of sleep among school-aged children during a school week. Sleep Disord. 2014;2014:315808. https://doi.org/10.1155/2 014/315808

45. Poulain T, Vogel M, Buzek T, Genuneit J, Hiemisch A, Kiess W. Reciprocal longitudinal associations between Adolescents' Media consumption and sleep. Behav Sleep Med. 2019:17:6, 763-777. https://doi.org/10.1080/15402 002.2018.1491851.

46. Scott H, Biello SM, Woods HC. Social media use and adolescent sleep patterns: cross-sectional findings from the UK millennium cohort stud. BMJ Open. 2019;9(9):e031161. https://doi.org/10.1136/bmjopen-2019-031161.

47. van der Schuur WA, Baumgartner SE, Sumter SR, Valkenburg PM. Media multitasking and sleep problems: a longitudinal study among adolescents. Comput Hum Behav. 2018;81:316-24. https://doi.org/10.1016/j.chb.2017.12. 024.

48. van der Schuur WA, Baumgartner SE, Sumter SR. Social media use, social media stress, and sleep: examining cross-sectional and longitudinal relationships in adolescents. Health Commun. 2019;34(5):552-9. https://doi. org/10.1080/10410236.2017.1422101.

49. Wallenius M, Rimpela A, Punamaki RL, Lintonen T. Digital game playing motives among adolescents: relations to parent-child communication, school performance, sleeping habits, and perceived health. J Appl Dev Psychol. 2009;30(4):463-74. https://doi.org/10.1016/j.appdev.2008.12.021.

50. Das-Friebel A, Perkinson-Gloor N, Brand S, Dewald-Kaufmann JF, Grob A, Wolke $\mathrm{D}$, et al. A pilot cluster-randomised study to increase sleep duration by decreasing electronic media use at night and caffeine consumption in adolescents. Sleep Med. 2019;60:109-15. https://doi.org/10.1016/j.sleep.201 8.11.010.

51. Perrault AA, Bayer L, Peuvrier M, Afyouni A, Ghisletta P, Brockmann C, et al. Reducing the use of screen electronic devices in the evening is associated with improved sleep and daytime vigilance in adolescents. Sleep. 2019;08:08.

52. Xu H, Wen LM, Hardy LL, Rissel C. Associations of outdoor play and screen time with nocturnal sleep duration and pattern among young children. Acta Paediatrica Int J Paediatrics. 2016;105(3):297-303. https://doi.org/1 0.1111/apa.13285.

53. Redmayne M, Smith E, Abramson MJ. The relationship between adolescents' well-being and their wireless phone use: A cross-sectional study. Environmental Health: A Global Access Science Source. 2013;12:90. http://dx. doi.org.proxy3-bib.sdu.dk:2048/10.1186/1476-069X-12-90

54. Vernon L, Barber BL, Modecki KL. Adolescent problematic social networking and school experiences: the mediating effects of sleep disruptions and sleep quality. Cyberpsychol Behav Soc Netw. 2015;18(7):386-92. https://doi. org/10.1089/cyber.2015.0107

55. Vernon L, Modecki KL, Barber BL. Tracking effects of problematic social networking on adolescent psychopathology: the mediating role of sleep disruptions. J Clin Child Adolesc Psychol. 2017:46(2):269-83. https://doi.org/1 0.1080/15374416.2016.1188702.

56. Zhang Z, Sousa-Sá E, Pereira J, Chaput J-P, Okely A, Feng X, et al. Correlates of nocturnal sleep duration, nocturnal sleep variability, and nocturnal sleep problems in toddlers: results from the GET UP! Study Sleep Med. 2019;53: 124-32. https://doi.org/10.1016/j.sleep.2018.08.035.

57. Ribner AD, McHarg GG. Why won't she sleep? Screen exposure and sleep patterns in young infants. Infant Behav Dev. 2019:57:101334.

58. Nuutinen $T$, Roos E, Ray C, Villberg J, Välimaa R, Rasmussen M, et al. Computer use, sleep duration and health symptoms: a cross-sectional study of 15-year olds in three countries. Int J Public Health. 2014;59(4):619-28. https://doi.org/10.1007/s00038-014-0561-y.

59. Vandendriessche A, Ghekiere A, Van Cauwenberg J, De Clercq B, Dhondt K, Desmet $A$, et al. Does sleep mediate the association between school pressure, physical activity, screen time, and psychological symptoms in early adolescents? A 12-country study. Int J Environ Res Public Health. 2019;16(6): 1072

60. Moorman JD, Morgan P, Adams TL. The implications of screen media use for the sleep behavior of children ages 0-5: a systematic review of the literature. Curr Sleep Med Rep. 2019;5(3):164-72. https://doi.org/10.1007/s4 0675-019-00151-0.

61. Carter B, Rees P, Hale L, Bhattacharjee D, Paradkar MS. Association between portable screen-based media device access or use and sleep outcomes. JAMA Pediatr. 2016;170(12):1202-8. https://doi.org/10.1001/jamapediatrics.2 016.2341.

62. Green A, Cohen-Zion M, Haim A, Dagan Y. Evening light exposure to computer screens disrupts human sleep, biological rhythms, and attention abilities. Chronobiol Int. 2017;34(7):855-65. https://doi.org/10.1080/0742052 8.2017 .1324878

63. Yau JC, Sun BT, Moreno JD. Addicting content, blue light, and curtailed sleep: the ABCs of social media use and sleep. The Psychology and Dynamics Behind Social Media Interactions: IGI Global. 2020:211-240. 
64. Baglioni C, Spiegelhalder K, Lombardo C, Riemann D. Sleep and emotions: a focus on insomnia. Sleep Med Rev. 2010;14(4):227-38. https://doi.org/10.101 6/j.smrv.2009.10.007.

65. Bartel KA, Gradisar M, Williamson P. Protective and risk factors for adolescent sleep: a meta-analytic review. Sleep Med Rev. 2015;21:72-85. https://doi.org/10.1016/.smrv.2014.08.002.

66. Mac Cárthaigh S, Griffin C, Perry J. The relationship between sleep and problematic smartphone use among adolescents: a systematic review. Dev Rev. 2020;55:100897. https://doi.org/10.1016/j.dr.2020.100897.

67. Guglielmo D, Gazmararian JA, Chung J, Rogers AE, Hale L. Racial/ethnic sleep disparities in US school-aged children and adolescents: a review of the literature. Sleep health. 2018;4(1):68-80. https://doi.org/10.1016/j.sleh.201 7.09.005.

68. Lin QM, Spruyt K, Leng Y, Jiang YR, Wang GH, Dong SM, et al. Cross-cultural disparities of subjective sleep parameters and their age-related trends over the first three years of human life: a systematic review and meta-analysis. Sleep Med Rev. 2019:48:101203. https://doi.org/10.1016/j.smrv.2019.07.006.

69. Chung SJ, An H, Suh S. What do people do before going to bed? A study of bedtime procrastination using time use surveys. Sleep. 2019,43:4, zsz267.

70. Eggermont S, Van den Bulck J. Nodding off or switching off? The use of popular media as a sleep aid in secondary-school children. J Paediatr Child Health. 2006;42(7-8):428-33. https://doi.org/10.1111/j.1440-1754.2006.00892. $x$.

71. Wolfson AR, Carskadon MA, Acebo C, Seifer R, Fallone G, Labyak SE, et al. Evidence for the validity of a sleep habits survey for adolescents. Sleep. 2003:26(2):213-6. https://doi.org/10.1093/sleep/26.2.213.

72. Short MA, Gradisar M, Gill J, Camfferman D. Identifying adolescent sleep problems. PLoS One. 2013;8(9):e75301. https://doi.org/10.1371/journal.pone. 0075301.

73. Short MA, Gradisar M, Wright H, Lack LC, Dohnt H, Carskadon MA. Time for bed: Parent-set bedtimes associated with improved sleep and daytime functioning in adolescents. Sleep. 2011;34(6):797-800. https://doi.org/10. 5665/SLEEP.1052.

74. Yetish G, Samson D. Sleep research in non-Western populations reveals novel insights about the breadth and diversity of human sleep patterns. Sleep Health. 2018;4(6):495-6. https://doi.org/10.1016/j.sleh.2018.10.006.

75. LeBourgeois MK, Hale L, Chang A-M, Akacem LD, Montgomery-Downs HE, Buxton OM. Digital media and sleep in childhood and adolescence. Pediatrics. 2017;140(Supplement 2):S92-S6.

\section{Publisher's Note}

Springer Nature remains neutral with regard to jurisdictional claims in published maps and institutional affiliations.

Ready to submit your research? Choose BMC and benefit from:

- fast, convenient online submission

- thorough peer review by experienced researchers in your field

- rapid publication on acceptance

- support for research data, including large and complex data types

- gold Open Access which fosters wider collaboration and increased citations

- maximum visibility for your research: over $100 \mathrm{M}$ website views per year

At BMC, research is always in progress.

Learn more biomedcentral.com/submissions 